\title{
Metschnikowia shivogae sp. nov., a yeast species associated with insects of morning glory flowers in East Africa
}

Correspondence

Marc-André Lachance

lachance@uwo.ca

\author{
Marc-André Lachance, ${ }^{1}$ Jane M. Bowles, ${ }^{1}$ T. Michael Anderson ${ }^{2,3}$ \\ and William T. Starmer ${ }^{2}$ \\ ${ }^{1}$ Department of Biology, University of Western Ontario, London, Ontario N6A 5B7, Canada \\ ${ }^{2}$ Department of Biology, Syracuse University, Syracuse, NY 13244, USA \\ ${ }^{3}$ Community and Conservation Ecology Group, University of Groningen, PO Box 14, 9750 AA \\ Haren, The Netherlands
}

\begin{abstract}
The novel species Metschnikowia shivogae is described to accommodate three isolates recovered from insects of morning glory flowers at two localities in East Africa. The isolates differ slightly in rDNA ITS and D1/D2 large-subunit sequences and one isolate featured a two-base heterogeneity that might be the result of recombination between two variant rDNAs. M. shivogae is a sister species to Metschnikowia aberdeeniae and shares the same habitat. The reproductive boundaries of $M$. aberdeeniae, which were not clear in the past, have now been elucidated further. The type strain of Metschnikowia shivogae sp. nov. is strain SUB 04-310.1 $1^{\top}\left(h^{+} ;=\mathrm{CBS}\right.$ $10292^{\top}=$ NRRL Y-27924 $\left.4^{\top}\right)$ and the allotype is strain UWOPS 07-203.2 $\left(h^{-} ;=\right.$CBS 10770 $=$ NRRL Y-48447).
\end{abstract}

The yeast species Metschnikowia aberdeeniae was described on the basis of five strains isolated from insects of flowers of morning glory (Ipomoea hildebrandtii, Convolvulaceae) in sites located near the Serengeti National Park, Tanzania (Lachance et al. 2006). This species is part of a growing clade of yeasts that form large needle-shaped ascospores and that occur with high frequency worldwide in nitidulid beetles and other insects that visit ephemeral flowers. The large-spored Metschnikowia species are haplontic and heterothallic, such that ascus formation must be preceded by conjugation between genetically compatible, independent isolates. Six strains isolated with $M$. aberdeeniae were related to the species, but their reproductive boundaries were uncertain. Two were identical to the type in their ITS/ $5.8 \mathrm{~S}$ and D1/D2 large subunit (LSU) rDNA sequences (sequence type A). Three differed by three substitutions (type B) and one by many more (about $8.6 \%$; type C). All these strains were of mating type $h^{+}$and, when crossed with the type of M. aberdeeniae $\left(h^{-}\right)$, produced mixtures of sterile and single-spored asci. As $h^{-}$strains of the divergent biotypes were not available, within-group fertility could not be assessed. Recently, additional isolates in the same clade have been recovered in the vicinity of Kabarnet, Kenya, from small clavicorn beetles collected in morning glory flowers. Eight were assigned to M. aberdeeniae and

The GenBank/EMBL/DDBJ accession numbers for the rDNA sequences of strains SUB 04-310.1 ${ }^{\top}$, UWOPS 07-204.1 and UWOPS 07-203.2 are respectively DQ266431, EU220026 and EU220027. two were found to be conspecific with the divergent isolate from Tanzania. We now describe the latter as Metschnikowia shivogae, named in honour of the family of William Shivoga, Dean of the Faculty of Environment and Resources Development of Egerton University, Egerton, Kenya. Professor Shivoga and his family went far beyond the call of duty in the process of collecting the new yeast strains discussed in this paper.

\section{Isolation and characterization}

The strains used in this study are listed in Table 1. Collection details for the Tanzanian isolates were given by Lachance et al. (2006). The Kenyan collection was conducted at two nearby locations along the road to Cheremon Field Station of Egerton University, Marigat, which is located about $265 \mathrm{~km}$ north-west of Nairobi, near the town of Kabarnet. The sites were separated by $400 \mathrm{~m}$. Ten samples were plated on acidified YM agar by allowing the insect to walk over the agar for 5-10 min. Three of the samples consisted of the meloid beetle Mylabris tristigma and gave rise only to unidentified moulds. The remainder were small clavicorn beetles tentatively assigned to the Nitidulidae. Drosophilids were also present in the same flowers. All yeasts were 'barcoded' by PCR amplification and rDNA sequencing as described previously (Lachance et al. 2005). Morphology and physiology were characterized by standard methods (Yarrow 1998). Mating reactions were obtained by mixing actively growing cells in pairs on 
Table 1. Selected properties of Metschnikowia isolates from East Africa

The accession numbers are those in the yeast culture collection of the Department of Biology, Syracuse University, Syracuse, NY, USA (SUB), the culture collection of the Department of Biology, University of Western Ontario (UWOPS), the Centraalbureau voor Schimmelcultures, Utrecht,

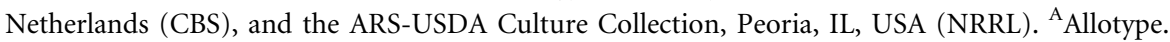

\begin{tabular}{|c|c|c|c|}
\hline Strain & Mating type & Source & Sequence type $\dagger$ \\
\hline \multicolumn{4}{|l|}{ Metschnikowia aberdeeniae } \\
\hline UWOPS 07-202.1 & $h^{+}$ & Kenya, 2007 & A \\
\hline UWOPS 07-202.3 & $h^{+}$ & Kenya, 2007 & A \\
\hline UWOPS 07-203.1 & $h^{-}$ & Kenya, 2007 & A \\
\hline UWOPS 07-204.2 & $h^{+}$ & Kenya, 2007 & A \\
\hline UWOPS 07-205.1 & $h^{-}$ & Kenya, 2007 & A \\
\hline UWOPS 07-207.1 & $h^{+}$ & Kenya, 2007 & A \\
\hline UWOPS 07-207.2 & $h^{+}$ & Kenya, 2007 & A \\
\hline UWOPS 07-208.1 & $h^{+}$ & Kenya, 2007 & A \\
\hline SUB $04-304.1^{\star}$ & $h^{+}$ & Tanzania, 2004 & A \\
\hline SUB $05-213.1^{\mathrm{T}}=\mathrm{CBS} 10289^{\mathrm{T}}=$ NRRL Y-2792 $1^{\mathrm{T}}$ & $h^{-}$ & Tanzania, 2005 & A \\
\hline SUB $05-213.2^{\mathrm{A}}=\mathrm{CBS} 10290^{\mathrm{A}}=\mathrm{NRRL} Y-27922^{\mathrm{A}}$ & $h^{+}$ & Tanzania, 2005 & A \\
\hline \multicolumn{4}{|l|}{ Metschnikowia shivogae sp. nov. } \\
\hline SUB $04-310.1^{\mathrm{T}}=\mathrm{CBS} 10292^{\mathrm{T}}=$ NRRL Y-27924 ${ }^{\mathrm{T}}$ & $h^{+}$ & Tanzania, 2004 & $\mathrm{C} 1$ \\
\hline UWOPS $07-203.2^{\mathrm{A}}=\mathrm{CBS} 10770^{\mathrm{A}}=\mathrm{NRRL} \mathrm{Y}-48447^{\mathrm{A}}$ & $h^{-}$ & Kenya, 2007 & $\mathrm{C} 2$ \\
\hline UWOPS 07-204.1 & $h^{+}$ & Kenya, 2007 & C3 \\
\hline
\end{tabular}

${ }^{\star}$ Species assignment previously uncertain (Lachance et al. 2006).

$†$ Sequence types A, B and C were defined by Lachance et al. (2006) and refer to the ITS/5.8S and LSU D1/D2 rDNA regions. Subtypes C1, C2 and C3 were identified in this study (see text for details).

yeast carbon base agar supplemented with $0.01 \%$ ammonium sulphate. Asci were detected at low magnification 1 day after mixing. The presence of ascospores was assessed after 2-3 days at higher magnifications. The strains were preserved in liquid nitrogen in the UWOPS yeast collection, Department of Biology, University of Western Ontario, and suitable vouchers were deposited in the collection of the Yeast Division of the Centraalbureau voor Schimmelcultures, Utrecht, Netherlands, and the ARS (NRRL) Culture Collection, Peoria, IL, USA, as indicated.

\section{Species delineation}

None of the Kenyan isolates had rDNA sequences of type B (Lachance et al. 2006), so it was not possible to improve our understanding of sexual fertility within that phylotype. The eight strains with type A sequences included representatives of both mating types. They were mixed in all possible combinations with the type and allotype of $M$. aberdeeniae, as well as strain SUB 04-304.1. The latter had been provisionally excluded from the species on the basis of the formation of mostly sterile asci. In this new experiment, we found that the proportion of asci containing two spores (as opposed to one or no spore) varied more as a function of individual strains and did not appear to be correlated with pairwise compatibility. Lachance \& Bowles (2004) found the ability to produce a substantial proportion of two-spored asci to be a good estimate of spore viability in other large-spored Metschnikowia species.
We therefore recognize all strains with sequence type A as members of $M$. aberdeeniae. The Kenyan strains UWOPS 07-203.2 and UWOPS 07-204.1 were similar but not identical in ITS/5.8S and D1/D2 LSU rDNA sequences to the Tanzanian strain SUB 04-310.1 ${ }^{\mathrm{T}}$ (type $\mathrm{C}$ sequences). The three strains were mixed in all possible reciprocal combinations and with the type and allotype of $M$. aberdeeniae. Abundant two-spored asci were found in crosses between the strains with type $\mathrm{C}$ sequences, but crosses with the other two strains (type A) gave rise only to sterile asci interspersed with a few single-spored asci. On this basis, we conclude that the three type $\mathrm{C}$ strains represent a distinct species, which we name Metschnikowia shivogae sp. nov.

Some of the sequence differences among the three strains assigned to M. shivogae are of special interest. In the ITS1 region, sequences $\mathrm{C} 1$ and $\mathrm{C} 3$ have the doublets TT and GA, respectively, whereas sequence $\mathrm{C} 2$ has both (KW), indicating a possible recombination event involving the ribosomal repeats. In the ITS2 region, sequence $\mathrm{C} 1$ differs from the other two by one substitution and one insertion. In the D1 domain, it is sequence C3 that differs from the other two, by one substitution. Finally, the D2 domain of sequence $\mathrm{C} 2$ differs from the other two, also by one substitution. The topology of the tree presented in Fig. 1 reflects some of these differences. Additional sampling over a larger geographical area should yield interesting data as to the significance of rDNA polymorphisms in M. shivogae. 


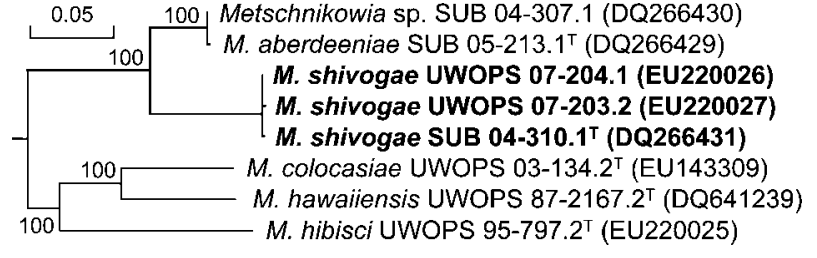

Fig. 1. Neighbour-joining phylogram depicting the relationships among strains of $M$. shivogae sp. nov. and related taxa based on ITS/5.8S and D1/D2 LSU rDNA sequences. The tree was rooted by the equal distance method. Bootstraps from 1000 pseudoreplicates with values of $50 \%$ or above are shown.

\section{Morphology, physiology and identification}

The cells and asci (Fig. 2) of M. shivogae are somewhat similar to those of M. aberdeeniae, but tend to resemble most those of species in the Metschnikowia arizonensis clade (Lachance \& Bowles 2002, 2004; Lachance et al. 2006). This is of interest for the following reason. The floricolous beetle-associated largespored Metschnikowia clade consists of four subclades, one of which contains the two African species. M. arizonensis and a few other species found in various Neotropical regions form another subclade (represented in Fig. 1 by Metschnikowia colocasiae). The largest subclade includes Metschnikowia hawaiiensis and a dozen close relatives. Last is a single species of Australian origin, Metschnikowia hibisci. Members of the M. hawaiiensis subclade tend to form the largest asci, with very long ribbon-like ascospores and a significant amount of periplasm, similar to what is seen in $M$. aberdeeniae. It therefore comes as a surprise that the asci of M. shivogae would instead resemble those found in the $M$. arizonensis clade, which are not as large and where much of the ascus volume is occupied by rather fusiform ascospores.

The growth responses are typical of most Metschnikowia species, although growth on some media is less vigorous. Specifically, growth on galactose, $\beta$-glucosides, mannitol, glucitol and D-glucosamine as carbon sources or lysine as nitrogen source, as well as glucose fermentation, were weaker compared with $M$. aberdeeniae. These differences are unsuitable for unambiguous identification, which should rely on mating tests or DNA sequencing.

\section{Latin diagnosis of Metschnikowia shivogae Lachance, Bowles et Starmer sp. nov.}

In medio YM post dies tres cellulae singulae, binae, aut in catenis brevis, ovoidae aut elongatae $(2-3 \times 4-9 \mu \mathrm{m})$. Cultura in agaro malti post dies 14 infima-convexa tumulosaque, glabra aut nitens, candida et butyrosa. In agaro carbonis fundamento post dies 14 pseudomycelium nec mycelium verum non-formatur. Post dies unus, cellulae stirpum interfertilium mixtarum in agaro carbonis fundamento tubi junctionis formantur. Post dies tres, magni asci cylindrati possunt videri. Asci stabiles sunt. Ascosporae aculeatae $(1.5-2 \times 45-60 \mu \mathrm{m})$. Glucosum (exigue) fermentatur. Glucosum, sucrosum, galactosum (exigue), trehalosum, maltosum, melezitosum, cellobiosum (exigue), salicinum (exigue), L-sorbosum, D-xylosum, ethanolum (exigue), glycerolum (lente), xylitolum, mannitolum (exigue), glucitolum (exigue), acidum succinicum (exigue), 2-ketogluconatum (lente) et $\mathrm{N}$-acetylglucosaminum assimilantur, at noninulinum, raffinosum, melibiosum, lactosum, methyl- $\alpha-\mathrm{D}-$ glucosidum, amylum solubile, L-rhamnosum, L-arabinosum, D-arabinosum, D-ribosum, methanolum, 1-propanolum, 2propanolum, 1-butanolum, erythritolum, ribitolum, galactitolum, meso-inositolum, acidum lacticum, acidum citricum, acidum gluconicum, glucono- $\delta$-lactonum, glucosaminum, acetonum, ethyl acetas nec hexadecanum. Ethylaminum, lysinum (exigue) et cadaverinum assimilantur at nonnatrium nitricum nec natrium nitrosum. Ad crescentiam vitaminae externae necessariae sunt. Augmentum in $32{ }^{\circ} \mathrm{C}$, at non $34{ }^{\circ} \mathrm{C}$. Habitat insectos e floribus Ipomoea spp. in Tanzania et Kenya. Typus SUB $04-310.1^{\mathrm{T}}\left(h^{+}\right)$. Allotypus UWOPS $07-203.2\left(\mathrm{~h}^{-}\right)$. In collectione zymotica Centraalbureau voor Schimmelcultures, Trajectum ad Rhenum, sub no. CBS $10292^{\mathrm{T}}$ et CBS 10770 depositae sunt.

\section{Description of Metschnikowia shivogae Lachance, Bowles et Starmer sp. nov.}

Metschnikowia shivogae (shi.vo'gae. N.L. gen. masc. sing. n. shivogae of Shivoga, in honour of William Shivoga and his family, in recognition of their contribution to the discovery of this species).

On YM agar after 3 days at $25{ }^{\circ} \mathrm{C}$, cells are ovoid to elongate, occur singly, in parent-bud pairs or in short

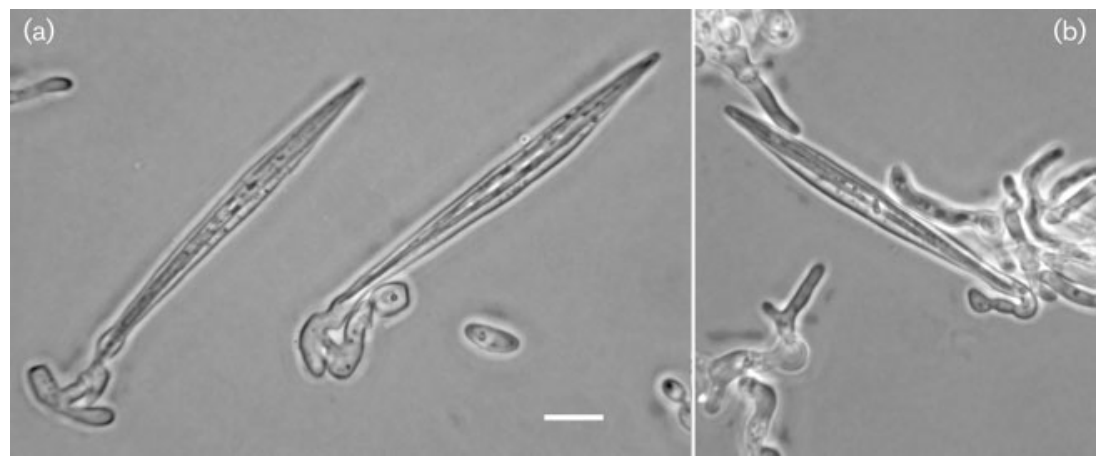

Fig. 2. Phase-contrast micrographs of asci of Metschnikowia shivogae after 2 days on YCBAS agar resulting from crosses between strains SUB $04-310.1^{\top}$ and UWOPS 07203.2 (a) and strains UWOPS 07-204.1 and UWOPS 07-203.2 (b). Bar, $10 \mu \mathrm{m}$. 
chains and measure 2-3 $\times 4-9 \mu \mathrm{m}$. After 2 weeks, colonies are low-convex and umbonate, semi-glossy to glossy, white and butyrous. In Dalmau plate culture on yeast carbon base agar after 2 weeks, pseudohyphae or true hyphae are not formed. On yeast carbon base agar at $18{ }^{\circ} \mathrm{C}$, mixtures of cells of complementary mating types give rise to zygotes and asci after $12-16 \mathrm{~h}$. After 3 days, mature asci containing two acicular ascospores $(1.5-2 \times 45-60 \mu \mathrm{m})$ are formed in a persistent ascus that retains conspicuous vestiges of the conjugated parent cells (Fig. 2). Fermentation: in glucose, a small amount of gas develops after 10-12 days. Glucose, sucrose, galactose (weak), trehalose, maltose, melezitose, cellobiose (very weak), salicin (weak), sorbose, xylose, ethanol (very weak), glycerol (slow), xylitol, mannitol (weak), glucitol (weak), succinic acid (weak), 2-ketogluconic acid (slow) and $\mathrm{N}$-acetyl-D-glucosamine are assimilated, but not inulin, raffinose, melibiose, lactose, methyl $\alpha$ D-glucoside, starch, L-rhamnose, L-arabinose, D-arabinose, D-ribose, methanol, 1-propanol, 2-propanol, 1-butanol, erythritol, ribitol, galactitol, inositol, lactic acid, citric acid, gluconic acid, glucono- $\delta$-lactone, D-glucosamine, acetone, ethyl acetate or $\mathrm{n}$-hexadecane. Ethylamine, lysine (weak) and cadaverine are utilized as nitrogen sources, but not sodium nitrate or nitrite. No growth in the absence of vitamins. Grows in the absence of amino acids. Grows at $32{ }^{\circ} \mathrm{C}$ but not at $34{ }^{\circ} \mathrm{C}$. Gelatin and casein hydrolysis is negative. Hydrolysis of Tween 80 is positive. Acid production on chalk agar is negative. Grows in the presence of $10 \% \mathrm{NaCl}$ but not $15 \%$. Growth in the presence of $50 \%$ glucose is slow. Growth in the presence of $10 \mathrm{mg}$ cycloheximide $1^{-1}$ is negative. Growth in the presence of $75 \mathrm{mg} \mathrm{CTAB}{ }^{-1}$ is positive. Starch production and diazonium blue $\mathrm{B}$ reaction are negative.

The known habitat is in insects associated with flowers of Ipomoea spp. in Tanzania and Kenya. The type strain is strain SUB $04-310.1^{\mathrm{T}}\left(h^{+}\right)\left(=\mathrm{CBS} 10292^{\mathrm{T}}=\right.$ NRRL Y$\left.27924^{\mathrm{T}}\right)$, recovered from a specimen of the meloid beetle Mylabris tristigma collected in Mugumu, Tanzania, near the Serengeti National Park. The allotype is strain UWOPS
07-203.2 $\left(h^{-}\right)(=\mathrm{CBS} 10770=\mathrm{NRRL}$ Y-48447), isolated from a clavicorn beetle collected near Kabarnet, Kenya. The name is registered under MycoBank number MB 511466 .

\section{Acknowledgements}

We acknowledge funding from the Natural Science and Engineering Council of Canada (M.-A. L.) and the National Science Foundation (W.T.S.). Isolation of strains in Kenya was facilitated by a memorandum of agreement between the University of Western Ontario and Egerton University, as well as funding from the Schulich School of Medicine Ecosystem Health Program. The cooperation of Mark Ritchie is gratefully acknowledged. Thanks are extended to William Shivoga and his family (Jacob, Winnie, Derek and Anne) as well as Eric Enanga and Irena Creed for field assistance. We thank James Kamal, Diana Lawrie and Jessica Dobson for laboratory assistance.

\section{References}

Lachance, M. A. \& Bowles, J. M. (2002). Metschnikowia arizonensis and Metschnikowia dekortorum, two new large-spored yeast species associated with floricolous beetles. FEMS Yeast Res. 2, 81-86.

Lachance, M. A. \& Bowles, J. M. (2004). Metschnikowia similis sp. nov. and Metschnikowia colocasiae sp. nov., two ascomycetous yeasts isolated from Conotelus spp. (Coleoptera: Nitidulidae) in Costa Rica. Stud Mycol 50, 69-76.

Lachance, M. A., Ewing, C. P., Bowles, J. M. \& Starmer, W. T. (2005). Metschnikowia hamakuensis sp. nov., Metschnikowia kamakouana sp. nov., and Metschnikowia mauinuiana sp. nov., three endemic yeasts from Hawaiian nitidulid beetles. Int J Syst Evol Microbiol 55, 13691377.

Lachance, M. A., Bowles, J. M., Anderson, T. M. \& Starmer, W. T. (2006). A new subclade of haplontic Metschnikowia species associated with insects of morning glory flowers in Africa and description of Metschnikowia aberdeeniae sp. nov. Int J Syst Evol Microbiol 56, 11411145.

Yarrow, D. (1998). Methods for the isolation and identification of yeasts. In The Yeasts, a Taxonomic Study, 4th edn, pp. 77-100. Edited by C. P. Kurtzman \& J. W. Fell. Amsterdam: Elsevier. 